Erschienen in: Stickel, Gerhard (Hrsg.): Deutsche Gegenwartssprache. Tendenzen und Perspektiven. - Berlin, New York: de Gruyter, 1990. S. 168-181. (Institut für deutsche Sprache. Jahrbuch 1989)

\title{
WALTER WEISS
}

\section{Sprache in der Literatur}

Das ist ein Rahmenthema, das mir wohl mit dem Blick auf meine vorwaltenden Interessen und auf meine Publikationen gestellt worden ist.

Ich bestimme es, mit dem Blick auf die Jubiläumstagung, näher als Rückbesinnung auf meinen Umgang mit dem Thema, in engerer und in weniger enger Verbindung mit dem Institut für deutsche Sprache, und auf die damit gekoppelten Veränderungen der Forschungssituation. Ich gehe dann etwas ausführlicher - anhand von Beispielen - auf mein augenblickliches Interessengebiet ein, das ich allerdings für zentral für die Frage nach der "Sprache in der Literatur" halte.

Dabei verstehe ich mich durch alle Wandlungen hindurch als ${ }_{n}$ Literaturwissenschaftler mit sprachwissenschaftlichen Interessen" als Grenzgänger, der dafür bei den eigentlichen Fachleuten um gütige Nachsicht bitten muß.

Es begann 1965 mit meinem 1967 in dem Band "Satz und Wort im heutigen Deutsch" publizierten Beitrag "Dichtung und Grammatik. Zur Frage der grammatischen Interpretation", ${ }^{1}$ worin ich mich für syntaktisch-semantische Beobachtungen an Texten von Kafka und Trakl der Wortklassen- und Satzteil-Kategorien der traditionellen Grammatik bediente, allerdings mit Einbeziehung der Arbeiten von Hans Glinz und Hennig Brinkmann.

Den nächsten Schritt tat ich mit dem Beitrag „Zur Stilistik der Negation" in der Festschrift für Hugo Moser $1969,{ }^{2}$ worin ich auf meinen vorangegangenen Untersuchungen zur „Negation im deutschen Satz" und zur "Verneinung mit un-" $(1960 / 1961)$ aufbaute. ${ }^{3}$ Dabei war ich mit dem "Paradigmenwechsel" zur gTG konfrontiert, von deren Basis aus Wolf Dietrich Hartung meinen syntaktisch-semantischen Ansätzen die radikale Trennung von "Oberflächenstruktur" und "Tiefenstruktur" entgegenhielt. ${ }^{4}$ Ich beharrte demgegenüber darauf, daß sich in der Negationsdiskussion von Philosophen und in der Dichtersprache zzumindest

1 Weiss 1967.

2 Weiss 1969.

3 Weiss 1961, 1960.

4 Hartung 1966. 
scheinbar Zusammenhänge zwischen [syntaktischer] Oberfäche und [logischer] Tiefe" (S. 265), ergeben und zeigte das an Beispielen von Hegel, Feuerbach, Heidegger, aus den "Nachtwachen" von Bonaventura, von Musil, Lessing, Karl Kraus, Kafka, Hölderlin, Goethe, Broch, Nestroy, Lenau.

Die geringe Eignung der gTG für Fragen und Phänomene der Literatursprache hat sich später auch an anderen Stellen gezeigt; sehr zum Schaden des versuchten Brückenschlags zwischen Sprach- und Literaturwissenschaft, die sich nun wieder entfremdeten.

Ein neues Hoffnungszeichen für eine mögliche Wiederannäherung setzten dann Textwissenschaft, Kommunikationswissenschaft, Semiotik, setzte die anivisierte Verbindung von Syntaktik, Semantik und Pragmatik. Im Anschluß daran versuchte ich im Rahmen des Münchner Gedenksymposions zu Thomas Manns 100. Geburtstag „Konkurrierende Ansätze sprachlicher Beschreibung und Deutung" an einem Text Thomas Manns ("Beim Propheten") zu erproben und in ihren Möglichkeiten gegeneinander abzuwägen. ${ }^{5}$ Ich wollte damit über mich selbst hinaus Literaturwissenschaftlern erneut Mut zur Wiederaufnahme abgebrochener interdisziplinärer Kontakte machen.

Besonders vielversprechend und anregend wurden für mich Harald Weinrichs Beiträge zur Metaphorik, die Metapher nicht mehr als "Wort" [oder Stilfigur], sondern als Stück Text in Textumgebungen und in textüberschreitenden Situationen (Kontexten) sehen lehrten. ${ }^{6}$

In der Zwischenzeit sind diese Perspektiven im großen und ganzen Allgemeingut geworden, wie der Forschungsbericht „zur sprachlichen Metaphorik" von Jürgen Nieraad (1977) und der Sammelband zur "Theorie der Metapher", herausgegeben von Anselm Haverkamp (1983), zeigen. ${ }^{7}$

Dabei fällt die lange tradierte Einschränkung der Metaphorik auf bestimmte Textsorten, wie rhetorische und literarische Texte im engeren Sinn, sie zeigt sich vielmehr über Textsortengrenzen hinaus sprachkonstitutiv. Weiter wird die überkommene Abwertung der Metaphorik als erkenntnishemmend und -störend problematisiert, wird sie als nicht ersetzbarer Gegenpol zum analytisch gewonnenen Erkennen, ja als erkenntnisleitendes Grundmodell für Paradigmen auch des wissenschaft-

\footnotetext{
5 Weiss 1977.

6 Weinrich 1976, S. 276-341.

7 Nieraad 1977; Haverkamp 1983.
} 
lichen Erkennens gewürdigt (so etwa Hans Blumenberg, ${ }^{8}$ Stichwort "Wissenschaftsmetaphorik").

Auf der anderen Seite wird zugleich mit dem Verzicht auf die von der linguistischen Poetik versuchte radikale Trennung (Opposition) von Literatursprache und Nichtliteratursprache - Hand in Hand mit einer Erweiterung des Literaturbegriffs, seiner Anwendung auch auf "Gebrauchstexte" - die Metaphorik als Kernbereich der Literatursprache neu herausgearbeitet. Ich habe dazu beigetragen, mit Titeln wie "Dichtersprache als Problem" (1974), „Anmerkungen zum Verhältnis von Sprach- und Literaturwissenschaft” (1985), „Von den Gleichnissen”, zu Kafka und Musil (1986), „Zur Metaphorik Th. Manns und R. Musils” (1985), „Th. Manns Metaphorik. Zwischenergebnis eines Forschungsprojekts" (1987). ${ }^{9}$

In der Folge möchte ich nun anhand der verteilten Texte mehr andeuten als ausführen, welche Gesichtspunkte im pauschal charakterisierten Neuansatz wirksam werden (können) und über Früheres hinausweisen. Der Gegenwartsbezug ergibt sich also mehr methodisch als in der Wahl der Beispiele. Im 2. Paar ist er allerdings gegeben, dort, wo der Text von Handke als ein Beispiel für literarische Wende behandelt und dem Text von Musil gegenübergestellt wird. Es zeigt sich dabei hoffentlich, daB hier ein Hoffnungsfeld für die Kooperation von Sprach- und Literaturwissenschaft liegt, und darüber hinaus auch für andere interdisziplinäre Kooperationen.

Die verteilten Texte bzw. Textausschnitte sind paarweise zu sehen:

1. Arthur Schnitzler: aus "Professor Bernhardi"10 und

2. Ludwig Wittgenstein, der Schluß des "Tractatus logicophilosophicus"; 11

3. Robert Musil „Die Maus" aus dem „Nachlaß zu Lebzeiten"12 und

4. Peter Handke "Laternen auf der Place Vendôme". ${ }^{13}$

8 Blumenberg 1957, 1960.

${ }^{\ominus}$ Weiss 1974, 1985, 1986, 1987.

${ }^{10}$ Schnitzler O.J., S. 446.

11 Wittgenstein 1965, S. 114/115.

12 Musil 1978.

13 Handke 1976. 
Arthur Schnitzler: Professor Bernhardi, Komödie in fünf Akten

FLINT Mein lieber Hofrat, wie ich privat zu diesen und anderen Fragen stehe, das ist ein Extrakapitel. So glattweg seine Ansichten daherplappern, das ist die Art politischer Dilettanten. Der Brustton der Überzeugung gibt einen hohlen Klang. Was wirkt, auch in der Politik, ist der Kontrapunkt.

HOFRAT Bis einer kommt, Exzellenz, dem wieder einmal eine Melodie einfällt.

FLINT Ganz fein. Aber um aus unserem metaphorischen Dialog wieder ins Reale hinabzusteigen, glauben Sie denn wirklich, lieber Hofrat, daß das Volk heute reif ist, oder jemals reif sein wird, ohne Religion zu existieren?

Beispiel (1) verrät ein traditionelles Metaphernverständnis, das auf der Gegenseite immer wieder auch zum „Kampf gegen die (eigentlich überflüssige) Metapher" stimuliert (hat): Metapher als begrifflich substituierbarer (ersetzbarer) Redeschmuck, den man zugunsten des „Realen" jederzeit vernachlässigen, weglassen kann.

Die Pointe liegt aber nun darin, daß der Wissenschaftsminister und Arzt Flint - er ist in der Tradition der Wiener Schule der Medizin um 1900 als naturwissenschaftlich ausgerichtet zu denken - tatsächlich gar nicht aus dem "metaphorischen Dialog" aussteigt, sondern nur metaphorisch umsteigt in das wissenschaftstheoretisch gegen das mechanistische Modell der Aufklärung eingesetzte bis politisch von der politischen Romantik, bis zum Nationalsozialismus und bis zu den heutigen Grünen überaus wirksame Modell (Paradigma) der Organismusmetaphorik: Stichwort reif. Dieses Modell spielte übrigens auch in Herrn Grosses Eröffnungsansprache über die Geschichte des IDS eine wichtige Rolle, dort, wo er davon sprach, daß das IDS und seine Planung in den 25 Jahren seines Bestehens "gewachsen" und "gereift" sei.

In der Ausdrucksweise von Anselm Haverkamps "Einleitung" zur "Theorie der Metapher":

Die metaphorische Situierung philosophischer [auch wissenschaftlicher W.W.] Texte in lebensweltlichen Bezügen, die ihnen hinter dem Rücken der Autoren in der aktuellen Lektüre zuwachsen, wird zunehmend in der literarischen Tradition von Texten der Moderne bewußt und interessant (S. 23).

Und in der griffigeren Ausdrucksweise des Antimetaphorikers RobbeGrillet:

Spricht man ... von einem in der Talmulde 'kauernden' Dorf ... Was ginge dem Dorf verloren, wenn es in der Talmulde 'gelegen' wäre? Das 
Wort 'gekauert' versetzt ... den Leser (im Anschluß an den Autor) in die vorgegebene Seele des Dorfes; wenn ich das Wort 'gekauert' gelten lasse, bin ich nicht mehr ein bloßer Zuschauer ... Fast in unserer ganzen zeitgenössischen Literatur wiederholen sich diese anthropomorphistischen Analogien viel $\mathrm{zu}$ beharrlich, viel zu kohärent, um nicht ein ganzes metaphysisches System [anders gesagt "Weltbild" oder ,ideologisches System", W.W.] zu verraten. ${ }^{14}$

Beispiel (2)

Ludwig Wittgenstein: Tractatus logico-philosophicus

Die Lösung des Problems des Lebens merkt man am Verschwinden dieses Problems. (Ist nicht dies der Grund, warum Menschen denen der Sinn des Lebens nach langen Zweifeln klar wurde, warum diese dann nicht sagen konnten, worin dieser Sinn bestand.)

Es gibt allerdings Unaussprechliches. Dies zeigt sich, es ist das Mystische. Die richtige Methode der Philosophie wäre eigentlich die: Nichto $z u$ sagen, als was sich sagen läßt, also Sätze der Naturwissenschaft also etwas, was mit Philosophie nichts zu tun hat -, und dann immer, wenn ein anderer etwas Metaphysisches sagen wollte, ihm nachzuweisen, daß er gewissen Zeichen in seinen Sätzen keine Bedeutung gegeben hat. Diese Methode wäre für den anderen unbefriedigend - er hätte nicht das Gefühl, daß wir ihn Philosophie lehrten - aber sie wäre die einzig streng richtige. Meine Sätze erläutern dadurch, daß sie der, welcher mich versteht, am Ende als unsinnig erkennt, wenn er durch sie - auf ihnen - über sie hinausgestiegen ist. (Er muß sozusagen die Leiter wegwerfen, nachdem er auf ihr hinaufgestiegen ist.)

Er muß diese Sätze überwinden, dann sieht er die Welt richtig.

Wovon man nicht sprechen kann, darüber muß man schweigen.

Beispiel (2) wird, vor allem mit seinem Schlußsatz, sehr viel, bis zum ÜberdruB, zitiert und greift die bereits im „Vorwort" proklamierte asketische Beschränkung auf ein-eindeutiges Sprechen und Denken, die Absage an jedes Sagen von „Metaphysischem” wieder auf. Dort im Vorwort heißt es:

Das Buch behandelt die philosophischen Probleme und zeigt - wie ich glaube - daß die Fragestellung dieser Probleme auf dem MiBverständnis der Logik der Sprache beruht. Man könnte den ganzen Sinn des Buches in die Worte fassen: Was sich überhaupt sagen läßt, läßt sich klar sagen; und wovon man nicht reden kann, darüber muB man schweigen. Das Buch will also dem Denken eine Grenze ziehen, oder vielmehr - nicht dem Denken, sondern dem Ausdruck der Gedanken ... Die Grenze wird also nur in der Sprache gezogen werden können und was jenseits der Grenze liegt, wird einfach Unsinn sein (S. 7).

14 Robbe-Grillet 1965. 
Ironischerweise - ob beabsichtigt oder nicht, bleibe dahingestellt - überschreitet nun aber Wittgenstein in der unmittelbaren Textumgebung die eben selbst gezogenen Grenzen metaphorisch. Das gilt bereits für die Aussage, in der wohl eine Exmetapher aktualisiert wird: „Es gibt allerdings Unaussprechliches. Dies zeigt sich, es ist das Mystische". Und dann für die zum ausgeführten Vergleich erweiterte Wendung: „- auf ihnen [den Sätzen] - über sie hinausgestiegen".

Die konstitutive (unverzichtbare) Rolle der Metaphorik wird da evident, wo sie sich, über die versuchten Abgrenzungen hinaus, auch gegen die erklärte Absicht der Sprachverwender, wieder einstellt.

[Ich habe darauf - weniger ausführlich als hier - in dem soeben (1988) erschienen Beitrag zur „Festschrift für Ingo Reiffenstein zum 60. Geburtstag" „Zur Tradition der Thematisierung der Sprache in der österreichischen Literatur" hingewiesen] . ${ }^{15}$

Den metaphorisch unterlaufenen Abgrenzungsversuchen zwischen metaphorischem und nichtmetaphorischem Ausdruck (Diskurs) stehen im 3. und 4. Beispiel "systematische" feldbildende metaphorische Grenzüberschreitungen, Kategorienmischungen, „Konterdeterminierungen” (nach Weinrich) gegenüber.

Das 3. Beispiel ist ausdrücklich im (1.) „Weltkrieg" situiert und steht in seiner 1. Fassung auch in Musils Kriegstagebuch. ${ }^{16}$

Beispiel (3)

Robert Musil: Die Maus

Diese winzige Geschichte, die eigentlich nur eine Pointe, eine einzige kleine Spitze ist, und gar keine Geschichte, ereignete sich im Weltkrieg. Auf der ladinischen Alpe Fodara Vedla, tausend und mehr Meter über bewohnter Gegend und noch viel weiter abseits von ihr: Dort hatte jemand im Frieden eine Bank hingestellt.

Diese Bank stand auch in Krieg unversehrt. In einer weiten, hellen Mulde. Die Schüsse zogen über sie hin. Ruhig wie Schiffe, wie Scharen von Fischen. Sie schlugen weit hinten ein, wo nichts und niemand war, und verwüsteten dort mit eiserner Beharrlichkeit seit Monaten einen unschuldigen Abhang. Niemand wußte mehr warum. Ein Irrtum der Kriegskunst? Eine Laune der Kriegsgötter? Diese Bank war dem Krieg in Verlust geraten. Und die Sonne schickte den ganzen Tag Licht aus unendlichen Höhen ihr zur Gesellschaft.

Wer auf dieser Bank saß, saß fest. Der Mund ging nicht mehr auf. Die

15 Weiss 1988 , S. $666 / 667$.

16 Musil 1976, S. 347. 
Glieder schliefen einen getrennten Schlaf wie Männer, die sich eng beisammen niedergeworfen und einander im gleichen Augenblick todmüd vergessen haben. Selbst das Atmen ward fremd; wurde ein Vorgang der Natur; nein, wurde nicht "Atem der Natur", sondern: wenn man bemerkte, daß man atme, - diese gleichmäßige, willenlose Bewegung der Brust! - etwas der Ohnmacht des Menschen vom blauen Ungeheuer Luft Angetanes wie eine Schwangerschaft.

Das Gras ringsum war noch vom Jahr vorher; schneebleich und häßlich; so blutleer, als ob man einen großen Stein davon weggewälzt hätte. In Nähe und Ferne gab es Buckel und Mulden ohne Sinn und Zahl, Knieholz und Alpe. Aus dieser bewegungslosen Unruhe, von dieser zu gelbgrünem Schaum zerfallenen Brandung des Bodens wurde der Blick immer wieder an dem hohen, roten Felsenriff emporgeworfen, das die Landschaft vorne abschloß, und rann, in hundert Blicke zersplittert, davon wieder ab. Es war nicht übermäßig hoch, dieses Felsstück, aber darüber war nur noch das leere Licht. So wïst war das und so un. menschlich herrlich wie in den Schöpfungszeitaltern.

Eine kleine Maus hatte sich nahe der Bank, die selten besucht wurde, ein System von Laufgräben angelegt. Maustief, mit Löchern zum Ver. schwinden und anderswo wieder aufzutauchen. Sie huschte darin im Kreise, stand, huschte im Kreis weiter. Aus dem Grollen der Luft tauchte eine ungeheure Stille auf. Die Menschenhand sank von der Lehne der Bank. Ein Auge, so klein und schwarz wie ein Spennadel. knopf richtet sich dahin. Und man hatte einen Augenblick lang ein so sonderbar verkehrtes Gefühl, daß man wirklich nicht mehr recht wußte, ob sich dieses kleine, lebendige, schwarze Auge drehe ader ob sich die ungeheure Unbeweglichkeit der Berge rühre. Man wußte nicht mehr: vollzog sich an einem der Wille der Welt oder der dieser Maus, der aus einem winzigen, einsamen Auge leuchtete. Man wußte nicht mehr: war Kampf oder herrschte schon Ewigkeit.

So hätte sich mit dem, was man nicht zu kennen fühlte, lange und nach Belieben fortfahren lassen; aber das ist schon die ganze kleine Ge. schichte, denn sie war inzwischen jedesmal schon zu Ende gegangen, ehe man noch genau sagen konnte, wo sie aufhörte.

Der Textsituierung entsprechend sind die im Text aufgebauten und ihn dominierenden Grenzüberschreitungen die zwischen ["Krieg"] ("Weltkrieg", "Kampf") und [Nichtkrieg], verbunden mit der Bank und der Maus ("auch im Krieg unversehrt", "dem Krieg in Verlust geraten", "Ewigkeit"). Das Wort- und Bildfeld des Krieges dringt aber auch ein in den Bereich des Nichtkrieges, so im Vergleich der Glieder des auf der Bank Sitzenden mit Männern, „die sich eng beisammen niedergeworfen", oder im „System der Laufgräben”, die die Maus angelegt hat. In der Gegenrichtung geraten Bilder des Nichtkrieges in den Bereich des Krieges, so im Vergleich für die über die Bank hinziehenden Schüsse: „Ruhig wie Schiffe, wie Scharen von Fischen". Dies bereitet auf die „Pointe" vor.

Ähnliches geschieht mit der Opposition von Weite und Enge, GröBe und Kleinheit, oben und unten, Mensch und Natur, Mensch und 
Tier, Lärm und "Stille”, „Unbeweglichkeit” und Beweglichkeit, bis zur „bewegungslosen Unruhe”. Da weiß man nicht mehr: „vollzog sich an einem der Wille der Welt oder der dieser Maus". Da vermischen sich die Bilder des Hochgebirges und des Meeres oder der Luft oben und des Meeres unten, verbunden mit der Vermischung von Lärm und Stille, in dem bereits zitierten Vergleich der Schüsse oben mit ruhig dahinziehenden Schiffen oder Fischen. Da verwandelt sich das Atmen des auf der Bank Sitzenden in einen "Vorgang der Natur", in ein "vom Ungeheuer Luft Angetanes", das dann wieder mit einer menschlichen "Schwangerschaft" verglichen wird. Da gehen ungeheure Unbeweglichkeit und (Kreis)bewegung der Berge, der Maus, des Mausauges bis zur Ununterscheidbarkeit ineinander über.

Die so metaphorisch vorbereitete "Pointe",,die einzige kleine Spitze" dieser "kleinen Geschichte" ist das im supponierten Subjekt situierte "sonderbar verkehrte Gefühl" der Unsicherheit, der Verwirrung, der Unentschiedenheit zwischen dem Kleinen (Maus, Mausauge) und dem Großen ("Berge", „Wille der Welt"), zwischen Beweglichkeit und Unbeweglichkeit, zwischen "Kampf" und "Ewigkeit”.

Und dies währt nur einen Augenblick, worin das Was und das Wie, der Inhalt und die Gestalt dieser „kleinen Geschichte”, die eigentlich "gar keine Geschichte ist", zusammenfallen.

Wir haben es hier also auch mit so etwas wie Formenmetaphorik zu tun, indem "diese winzige Geschichte" unabsehbar Großes in sich faßt. Auch formal gesehen verkehren sich Kleines und Großes, „winzige Geschichte” und Weltgeschichte, in der Opposition von "Kampf" und "Ewigkeit”.

In der Metaphorikforschung hat Susanne Ledanff die wichtige Rolle der „Augenblicksmetapher" in der (für die) Literatur des 20. Jahrhunderts herausgearbeitet. ${ }^{17}$

Ein Blick auf den Kontext dieser Geschichte im Werk Musils zeigt, daß sie ein besonders prägnantes Beispiel für den ${ }_{n}$ a.Z." (anderen Zustand) ${ }^{18}$ bietet, um den übrigens fast alle anderen Texte der Abteilung "Bilder" im „Nachlaß zu Lebzeiten (1936) kreisen, der „Die Maus” entnommen ist.

Das 4. Beispiel, die Kurzprosa von Handke, bietet auf den ersten Blick deutliche Entsprechungen zum Musil-Text.

17 Ledanff 1981.

18 Karthaus 1965. 
Peter Handke: Die Laternen auf der Place Vendôme

\begin{abstract}
Rund um den Platz Vendôme stehen die in Paris häufigen dreiarmigen Laternen. Ein Fußgänger, der seit langem getrennt war von denen, die er liebte, kam auf den Platz. Es dämmerte schon. Er hielt an und wollte auf einmal die Lichter angehen sehen. Ungeduldig umkreiste er den Platz in der einen, dann in der anderen Richtung, und sein Herz fing an zu klopfen, wie bei einem Abenteuer. Er hatte Angst, den Moment zu verpassen - zu blinzeln zum Beispiel, oder unwillkürlich in die Juweliergeschäfie zu schauen, wo auf Samthockern tief im Raum die Verkäufer saßen. Allmählich schlugen die Laternen in Zeichen um, und hinter ihrem blankgeputzten Glas trieben die stürmischen Wolken des Dämmerungshimmels über ein in die Unendlichkeit verzerrtes Todestal, von den eben Gestorbenen zu den nächsten Sterbenden - die Endoberfiäche sank mit dem Himmel unter den Lebensspiegel.

Endlich, während er in Panik weiter um den Platz herumging, flammten ringsum die Lichter auf. Sie wurden sofort ganz hell, in einem Augen. blick der starken Freude, in dem der Fußgänger die ewige Untröstlichkeit zugleich als Asyl erlebte. Zu Baden blickend, fühlte er sich bei dem schmutzigen Gehsteig jetzt heimisch. Er konnte weitergehen, endlich wieder geradeaus - nicht friedlich, aber in einer Heiterkeit, die auch wehtat. Der Himmel wurde blau, und der Abendstern erschien. Dieser kleine Stadthimmel erinnerte an nichts Bestimmtes mehr, wie es auch auf der windigen Brücke vom Fluß herauf nach nichts Bestimmtem rach. Nur daß er den Entgegenkommenden oft auf die falsche Seite auswich, war übriggeblieben von der Gewißheit, gerade noch gelebt zu haben: eine erstorbene Empfindung, die kalt durch sein Bewegungszentrum irrte.

Der Fußgänger folgte einer Verabredung und ließ sich betäuben von der auf ihn eindringenden Leibeigenen-Sprache, das Gesicht beim Zuhören noch nicht gelöst vom Krampf des Alleinseins. Aber später in der Nacht fing es plötzlich zu hageln an. Sehr helle Hagelkörner blitzten aus der Dunkelheit über der Straßenbeleuchtung und fielen schräg auf die trockenen, noch tagwarmen Gehsteige; schlitterten ein bißchen weiter und schmolzen, indem sie liegenblieben, sofort weg, ausgelöscht in dem starken Augenblick der Freude, in dem Stunden vorher die Laternen auf dem Platz Vendôme aufgeflammt waren.
\end{abstract}

$\mathrm{Da}$ ist zunächst einmal die Rolle des Augenblicks, des "Moments", hier als „ein Augenblick der starken Freude", aber auch die entscheidende Bedeutung dieses Augenblicks als „Pointe" der dargestellten Welt- und Ichverfassung.

Von da aus ergeben sich Querverbindungen sowohl zum a.Z. bei Musil wie auch zu zeitlich benachbarten Texten Handkes, in denen sich die vieldiskutierte, vielumstrittene "Wende" seiner Poetik von der sprachexperimentellen Konstruktion und Reproduktion zum ${ }_{\mu}$ Wiederho- 
len" 19 des klassisch Vorbildlichen, Gültigen, vom Kritischen zum Tröstlichen, von der Ablehnung des Vergleichens ${ }^{20}$ zur Wiedergeburt der Metapher ereignet. So erfährt etwa in "Die Stunde der wahren Empfindung" (1975) ${ }^{21}$ die verunsicherte, vereinsamte, von Todesangst umgetriebene Zentralfigur Gregor Keuschnig bei einbrechender Dämmerung auf einer Bank am Carré Marigny in Paris zuerst die "höllische Allerwelt", die "sich ein(richtete) wie für immer" (S. 78). Dann aber hat er ein verwandelndes Erlebnis. Drei unscheinbare Dinge, die schon die ganze Zeit so dagelegen hatten, „ein Kastanienblatt; ein Stück von einem Taschenspiegel; eine Kinderzopfspange" rücken "auf einmal" „zusammen zu Wunderdingen”, die ihm die Frage aufdrängen: „Wer sagte denn, daß die Welt schon entdeckt ist?” (S. 81) „Er erschrak ... vor Freude. Er fühlte sich auf einmal so befreit" (S. 82). Und im Rückblick darauf heißt es später deutend-zusammenfassend: „Dieser Zustand war keine Laune, kein Augenblickszustand mehr ... Indem ihm die Welt geheimnisvoll wurde, öffnete sie sich und konnte zurückerobert werden" (S. 152).

Die Entsprechung zu dem Augenblick bzw. zu den Augenblicken auf der Place Vendôme drängt sich auf. Neben den Entsprechungen zeigen sich nun aber auch ebenso deutliche Unterschiede zum Text Musils. Der "Augenblick" ist nicht einmalig, sondern er wiederholt sich: Zuerst das Auflammen der Lichter auf der Place Vendôme, dann das Aufblitzen der Hagelkörner im Licht der Platzbeleuchtung. Diese Augenblicke werden ausdrücklich gleichgesetzt. Das erfahrende Subjekt ist in der Er-Form (als Fußgänger) ausdrücklich markiert und nicht nur supponiert, wie in „Der Maus”. Der Betrachtergestus stimmt dagegen überein.

Und der Inhalt (die Semantik) dieser Augenblicke wird nicht bestimmt durch das Merkmal „verkehrt", enthält nicht die Aufhebung der sicheren, klaren Orientierung, sondern bringt im Gegenteil einen Orientierungsgewinn mit sich: "Er konnte weitergehen, endlich wieder geradeaus"; er erfährt darin die "Gewißheit, ... gelebt zu haben”.

Dem entspricht die Beobachtung, dabi sich die bedeutungstragenden Ausdrücke dieses Textes, besonders die metaphorischen, im Bezug auf das erfahrende Subjekt und auf die metasprachliche Formulierung: "Allmählich schlugen die Laternen in Zeichen um", zwanglos zwei Feldern zuordnen lassen, die durch die Merkmalopposition [negativ be-

19 Handke 1983, 1986.

20 Handke 1968.

21 Handke 1975. 
setzt, bewertet], vs. [positiv besetzt, bewertet] zu bestimmen ist, oder wenn man den Jargon der Kritiker Handkes gebraucht, durch [unheil] vs. [heil] .

negativ besetzt: stürmische Wolken des Dämmerungshimmels, von den eben Gestorbenen zu den nächsten Sterbenden, sank ... unter den Lebensspiegel, Panik, ewige Untröstlichkeit, schmutziger Gehsteig, erstorbene Empfindung, kalt ... irrte, betäuben, Leibeigenensprache, Krampf des Alleinseins.

positiv besetzt: Abenteuer, Lichter angehen, sofort ganz hell, Augenblick der starken Freude, Asyl, heimisch, Heiterkeit, Himmelblau, Abendstern erschien, sehr helle Hagelkörner blitzten, starker Augenblick der Freude, Laternen aufgeflammt.

Kombinationen aus dem oppositionellen Ausdrücken bzw. Merkmalen: ewige Untröstlichkeit zugleich als Asyl; schmutziger Gehsteig heimisch, Heiterkeit, die auch wehtat; ausgelöscht in dem ... starken Augenblick der Freude.

Dieser Befund provoziert erneut einen Vergleich mit Musils Text: Die größere Komplexität der metaphorischen Analogsetzungen und Vertauschungen dort steht der größeren Simplizität der Aufteilung in Handkes Text gegenüber.

Ich bin versucht, dies zugunsten Musils zu bewerten. Dabei muß ich mich aber selbstkritisch fragen, ob diese Wertung nicht zu tun hat mit einer vorgegebenen, vorentschiedenen Vorliebe für das Komplexe, Nichtgelöste, Offene, gegenüber dem betont Einfachen, der klaren „gültigen" Lösung.

Jedenfalls hat dieser Unterschied auch Folgen für die Rezeption; denn Musils Text verlangt vom Leser mehr kombinierende, weiterdenkende Produktivität als der Handkes. So ergibt sich ein Übergang von der vergleichenden Analyse (Beschreibung) zu einer Wertung, die ihre Kriterien offenlegt. Für den Literaturwissenschaftler ist eine solche Möglichkeit wichtig, wenn nicht unerläßlich.

Ich komme zum SchluB: Es war meine Absicht anzudeuten, daß die TextKontext-Metaphorik ein hoffnungsträchtiges interdisziplinäres Arbeitsfeld für Literatur- und Sprachforscher ist. Sie müssen entscheiden, ob bzw. wie weit mir das gelungen ist.

Eine letzte Bemerkung kann ich beim Blick auf die (rezenten) Veränderungen in der Theorie der Metapher nicht unterdrücken: Alles, was sich da an Neuem tut, geht doch immer noch zurück auf die bereits von 
Aristoteles eingeführten Kriterien der "Analogie" (Teilgleichheit, Teilidentität) und der „Übertragung" (oder auch Kombination, Verbindung, Integration). Jürgen Nieraad beschließt seinen Forschungsbericht dementsprechend:

Faktisch geht aber nahezu jede der referierten Arbeiten von einer in der Regel nicht explizierten Metaphernauffassung aus, wobei die aristotelische Übertragungsdefinition den großen Hut abgibt, unter der alles paBt (S. 111).

Zwei wichtige Veränderungen scheinen mir allerdings damit gegeben, daß die Substitutionstheorie (möglicher Ersatz der Metapher durch den "eigentlichen" Ausdruck) gefallen ist und daß die "Analogien" (Teilgleichheiten, Teilidentitäten) weniger als vorgefundene denn als gestiftete, gesetzte, hergestellte aufgefaßt werden.

\section{Literatur}

\section{Primärliteratur}

Handke, Peter (1968): Das Elend des Vergleichens. In: Prosa Gedichte Theaterstücke Hörspiele Aufsätze. Frankfurt am Main 1969, S. 314-326.

Handke, Peter (1975): Die Stunde der wahren Empfindung. Frankfurt am Main.

Handke, Peter (1976): Laternen auf der Place Vendôme. In: Zwischenbilanz. Eine Anthologie österreichischer Gegenwartsliteratur, hrsgg. von Walter Weiss und Sigrid Schmid. Salzburg, S. 34-35.

Handke, Peter (1983): Der Chinese des Schmerzes. Frankfurt am Main.

Handke, Peter (1983): Phantasien der Wiederholung. Frankfurt am Main (= edition suhrkamp 1168, Neue Folge 168).

Handke, Peter (1986): Die Wiederholung. Frankfurt am Main.

Musil, Robert (1976): Tagebücher. Reinbek bei Hamburg.

Musil, Robert (1978): Gesammelte Werke 7. Reinbek bei Hamburg.

Schnitzler, Arthur (o.J.): Die Dramatischen Werke. 2. Bd. Frankfurt am Main.

Wittgenstein, Ludwig (1965): Tractatus logico-philosophicus. Logisch-philosophische Abhhandlung. Frankfurt am Main (=edition suhrkamp 12). 


\section{Sekundärliteratur}

Blumenberg, Hans (1957): Licht als Metapher der Wahrheit. In: Studium generale 10 , S. 432-447.

Blumenberg, Hans (1960): Paradigmen zu einer Metaphorologie. In: Archiv für Begriffsgeschichte 6, S. 7-142, S. 301-305.

Haverkamp, Anselm (Hrsg.) (1983): Theorie der Metapher. Darmstadt (= Wege der Forschung Bd. 389).

Hartung, Wolf Dietrich (1966): Die Negation in der deutschen Gegenwartssprache. In: Deutsch als Fremdsprache 3, H. 2, S. 13-19.

Karthaus, Ulrich (1965): Der Andere Zustand. Zeitstrukturen im Werk Robert Musils. Berlin.

Ledanff, Susanne (1981): Die Augenblicksmetapher. München.

Nieraad, Jürgen (1977): „bildgesegnet und bildverflucht”. Forschungen zur sprachlichen Metaphorik. Darmstadt (= Erträge der Forschung Bd. 63).

Robbe-Grillet, Allain (1965): Argumente für einen neuen Roman. Essays. München.

Weinrich, Harald (1976): Sprache in Texten. Stuttgart.

Weiss, Walter (1960): Die Verneinung mit "un-". In: Muttersprache 70, S. 335-343.

Weiss, Walter (1961): Die Negation im deutschen Satz, I und II. In: Wirkendes Wort 11, S. 65-74, S. 129-140.

Weiss, Walter (1967): Dichtung und Grammatik. In: Satz und Wort im heutigen Deutsch. Düsseldorf, S. 236-258.

Weiss, Walter (1969): Zur Stilistik der Negation. In: Festschrift für Hugo Moser zum 60. Geburtstag. Düsseldorf, S. 263-281.

Weiss, Walter (1974): Dichtersprache als Problem. In: Sprachthematik in der österreichischen Literatur des 20. Jahrhunderts. Wien, S 27-39.

Weiss, Walter (1977): Konkurrierende Ansätze sprachlicher Beschreibung und Deutung. Angewendet auf die Erzählung „Beim Propheten” von Thomas Mann. In: Thomas Mann 1875-1975. Vorträge in München-ZürichLübeck. Frankfurt am Main, S. 484-499. 
Weiss, Walter (1985): Anmerkungen zum Verhältnis von Sprach- und Literaturwissenschaft. In: Literatur und Sprache im Österreich der Zwischenkriegszeit. Stuttgart, S. 257-265.

Weiss, Walter (1985): Zur Metaphorik Thomas Manns und Robert Musils. Text und Kontext. In: Jahrbuch für Internationale Germanistik XVII, H. 1, S. 58-76.

Weiss, Walter (1986): Von den Gleichnissen. In: Jahrbuch für Internationale Germanistik. Reihe A, Bd. 17, S. 128-139.

Weiss, Walter (1987): Thomas Manns Metaphorik. Zwischenergebnisse eines Forschungsprojekts. In: Internationales Thomas-Mann-Kolloquium 1986 in Lübeck. Bern, S. 311-326 (= Thomas-Mann-Studien VII).

Weiss, Walter (1988): Zur Tradition der Thematisierung der Sprache in der österreichischen Literatur. In: Festschrift für Ingo Reiffenstein zum 60. Geburtstag. Göppingen, S. 663-675. 